\title{
Characteristics of symptoms of imminent eclampsia: A case referent study from a tertiary hospital in Tanzania
}

\author{
John France, Projestine S. Muganyizi
}

Department of Obstetrics \& Gynecology, Muhimbili University of Health and Allied Sciences (MUHAS), Dar es Salaam, Tanzania Email: rwegoshorafrance@yahoo.co.uk, promuga@yahoo.com

Received 24 April 2012; revised 26 May 2012; accepted 10 June 2012

\begin{abstract}
Background: Maternal mortality in developing countries is unacceptably high with eclampsia being consistently among the top causes. As yet, primary prevention of this complication is not possible since causes of pre-eclampsia are largely unknown and biochemical, hematological and radiological markers have proved unsuitable for routine prediction of eclamptic fits. Although headache, visual disturbance, abdominal pain, nausea, and vomiting are routinely elicited when managing pre-eclampsia and have been reported to predict eclamptic fits, the literature attempting to characterize them is scanty. We sought to

The general occurrence of abdominal pain, nausea and vomiting was not significantly different in the two groups. The time lag from development of a symptom to eclamptic fit was up to seven days for most symptoms except visual disturbances of which $98 \%$ developed fits within 12 hours. Conclusion: Whereas the prodromal symptoms of eclampsia and similar symptoms in normotensive women were common, the characteristics of headache and visual disturbance differ significantly in the two groups. The knowledge of these differences could be utilized to improve the quality of management of pre-eclamptic women in order to prevent eclampsia.
\end{abstract} establish characteristics of the prodromal symptoms of eclampsia and compare them with similar symptoms as experienced by normotensive pregnant women at Muhimbili National Hospital (MNH) in Tanzania. Methods: This study was conducted at MNH in 2010 by enrolling 123 eclamptic and 123 normotensive women. Women in the two groups were interviewed about their experiences and characteristics of headache, visual disturbances, abdominal pain, nausea and vomiting using a semi structured questionnaire. The severity, nature and other characteristics of the symptoms were assessed using standard scale/methods and data compared among the two groups. Results: Prodromal symptoms of eclampsia were present in $\mathbf{9 0 \%}$ of eclamptic women. Headache was more frequent among eclamptic women $(88 \%)$ than the normotensive $(43 \%), p<0.001)$. The symptom was also more perceived as severe among eclamptic $(46.3 \%)$ than the normotensive $(5.7 \%), p<0.001$. The most frequent location for headache was frontal in $65.7 \%$ of eclamptic women compared to frontal $\mathbf{( 4 1 . 5 \% )}$ or generalized $(39.6 \%)$ for the normotensive. Likewise, visual problems were significantly more frequent among eclamptic women (39\%) compared to the normotensive $(3 \%), p<0.001$. Upper abdominal pain was significantly more reported by eclamptic $(36 \%)$ than normotensive women $(0.9 \%), p=0.001$.
Keywords: Eclampsia; Symptoms; Headache; Blurring of Vision; Abdominal Pain; Tanzania

Pre-eclampsia (PE) is a multisystem hypertensive disorder of unknown cause that is unique to human pregnancy. It is characterized by abnormal vascular response following placentation that leads to functional changes such as increased systemic vascular resistance, enhanced platelet aggregation, activation of coagulation system and endothelial cell dysfunction [1,2].

Symptoms that accompany pre-eclampsia are a result of generalized vasospasms, fibrin and platelet deposition and occlusion of blood flow to vital organs. In severe cases the liver is affected where sub capsular haemorrhage, necrosis and edema of the liver cell occurs producing epigastric pain and impaired liver function [3]. The brain becomes edematous and this in conjunction with vasospasm hypertension and disseminated intravascular coagulation (DIC) can produce cerebral under perfusion, ischaemia, and necrosis of blood vessel resulting in headaches, visual disturbances and cerebrovascular accident $[4,5]$.

Pre-eclampsia affects $5 \%$ to $10 \%$ of all pregnancies and is not confined to any population group [6,7]. Globally eclampsia accounts for $12 \%$ maternal mortality

\section{INTRODUCTION}


mostly in the developing countries where the incidence of eclampsia is high and quality of care of pre-eclamptic women is low [8-13]. The case fatality rate for clampsia is less than $1 \%$ in many developed countries and generally above $5 \%$ in developing countries [7,9-12].

The exact incidence of eclampsia in Tanzania is unknown and the few available data are hospital based. An estimated 67 cases of eclampsia occur per 10,000 births in Dar es Salaam community [14]. At Muhimbili National Hospital (MNH) where eclampsia is among the top two causes of maternal mortality the incidence of eclampsia ranges from 200 - 504 per 10,000 births with a case fatality rate of about $5 \%$ [14-16].

In spite of intense basic research, the etiology and pathophysiology of pre-eclampsia and eclampsia is still not fully understood $[17,18]$. Currently there are no reliable methods suitable for routine screening for both preeclampsia and eclampsia, hence primary prevention of pre-eclampsia is not possible. The validity of biochemical, hematological and radiological markers to predict eclampsia has been practically insufficient to recommend their routine use [13,18-23]. The absence of clear diagnostic markers to predict the risk of progression from pre-eclampsia into eclampsia has made researchers to investigate symptoms that can be used to predict the onset of eclampsia [21,24-26].

Headaches and visual symptoms, epigastric abdominal pain, nausea and vomiting have been consistently reported as important premonitory symptoms for eclamptic fits [24-26]. One systematic review has recently reported the usefulness of these symptoms in predicting complications of pre-eclampsia compared with when they are not present $[21,25]$, but others have cautioned on the reliance on such routine risk factors to predict maternal outcomes [27]. Although these symptoms are routinely elicited when managing pre-eclampsia patients, they are poorly defined. The few studies that have attempted to investigate premonitory symptoms have neither included detailed descriptions of the individual symptoms nor compared them with similar complaints in normotensive pregnant women [24]. Moreover, it is well known that the occurrence of neurological symptoms such as headache and gastrointestinal symptoms of nausea and vomiting may be exaggerated in normal pregnancy due to physiological changes [28-31]. Furthermore, in the tropics such symptoms might be confused with symptoms due to parasitic infections such as malaria of which pregnant mothers tend to be vulnerable [8,31]. Given this background the current study was designed in order to determine distinguishing clinical features of the prodromal symptoms of eclampsia. Our results were expected to contribute to improvement in the recognition and management of severe pre-eclampsia and prevention of eclampsia.

\section{METHODS}

\subsection{Study Settings}

This hospital based case referent study was conducted from April to August 2010 at MNH in Dar es Salaam, Tanzania. MNH is one of the four referral hospitals in Tanzania. It offers specialized obstetric services for Dar es Salaam city which is the biggest in Tanzania, and its suburbs. The city of Dar es salaam is estimated to have a population of 3.4 million people according to the 2002 national population census with an annual growth rate of 4.3\%. There are 3 districts in Dar es Salaam; Ilala, Temeke and Kinondoni. Each district has a district hospital which provides emergency obstetric care. However most of obstetric emergencies in Dar es Salaam are referred to $\mathrm{MNH}$. In addition patients are also referred from nearby regions. Apart from the referred patients, a substantial number of patients with or without obstetric complication come directly from home.

\subsection{Study Sample}

For the cases, the population constituted eclamptic women admitted in ICU at MNH with a diagnosis of Eclampsia. Eclampsia was defined according to the working protocol as development of generalized fits in a woman with a blood pressure of 140/90 $\mathrm{mmHg}$ or higher and proteinuria of + or higher on dipstick without any recognizable cause. Referents were normotensive women who sought maternity services (i.e. including mothers cared in labor ward or due to other obstetric reasons including routine antenatal clinic attendance) at $\mathrm{MNH}$ around the time of admission of a case to ICU.

All consecutive admissions to ICU with a diagnosis of eclampsia and who met the criteria were prospectively enrolled as cases until the desirable sample size was realized. For every enrolled eclamptic woman, one referent woman who best matched the case in terms of age, parity, gestation age, status and mode of delivery was identified. The matching variables corresponded to the status of the case at the onset of the fit. For example, if the fit occurred before labor, the match was a normotensive pregnant woman who is not in labor among mother who were seeking maternity services at around the time of admission of the patient and who made the best match with the rest of matching variables (i.e., age, parity, gestation age). Mothers were excluded from the study if they were of unsound mind, had developed eclampsia more than 72 hours after delivery, or could not communicate verbally.

In sample size estimation, we wished to compare the incidence of visual disturbance among eclamptic women with the referent women. The incidence of visual disturbance was taken as $32 \%$ for eclamptics. We hypothesized that the incidence among referent group of women would 
be lower, say $15 \%$. Thus the calculated minimum required sample size would be 216 , that is, 108 eclampsia and 108 referent women assuming 95\% confidence and power of $80 \%$ [32].

\subsection{Data Collection}

Data were collected using interviewer administered semi structured and checklist questionnaires. Information was obtained both directly from the women and from the case notes. Women who had experienced eclamptic fits were asked their experiences with the occurrence and characteristics of headache, visual disturbances, abdominal pain, nausea and vomiting preceding their fit(s).

\subsection{Data Analysis}

Data were coded and entered into computer using Epi data program. The software used for analysis was IBM SPSS statistics 19. Comparison of proportions used $x^{2}$ and Fischer exact test. Continuous or ordinal data were compared using a t-test with the assumption of equal variance.

In the analysis, the location of headache was described according to standard anatomical divisions of the head and its severity using a four grade scale (4GS). According to the 4GS pain severity was scored as; no pain (0), mild (1), moderate (3) and severe pain (4). The 4GS has been found to be as effective as the visual analogue scale (VAS) by other researchers [33]. Visual symptoms were characterized as blurring, blind sports, photophobia, and total blindness consistent with other studies [34-36]. Other symptoms include abdominal pain (type and location) and nausea/vomiting.

\section{RESULTS}

During the study period 130 eclamptic women were admitted, of which 7 did not fulfill the inclusion criteria. Thus, 123 were enrolled for the study as cases and were matched with 123 normotensive women (referents) to make up a total of 246 women for the study. For eclamptic women, age ranged from 16 to 37 years with a median of 22 years. The mean gestation age was 35.5 weeks and the mean parity was 2 . A total of, $83(68 \%)$ eclamptic women had delivered and 40 (32\%) had not delivered at the time of interview (Table 1).

Majority of eclamptic women (70\%) were between 20 35 years of age. Most of them were primipara (68\%), about half of them were at term.

As seen from Table 2, generally a significantly bigger proportion of eclamptic women presented with morbid symptoms $(90 \%)$ compared with normotensive mothers (54\%). Headache and visual problems in particular were significantly more frequent in eclamptics than in referent
Table 1. Characteristics of eclamptic and normotensive women. Data presented as n (\%).

\begin{tabular}{cccc}
\hline Characteristic & Eclamptic & Normotensive & Total \\
\hline Age (yrs) & & & \\
$<20$ & $36(29.0)$ & $35(29.0)$ & $71(29.0)$ \\
$20-35$ & $86(70.0)$ & $86(70.0)$ & $172(70.0)$ \\
$>35$ & $1(1.00)$ & $2(1.00)$ & $3(1.00)$ \\
Parity & & & \\
Primigravida & $82(67.0)$ & $85(69.0)$ & $167(68.0)$ \\
Multipara & $41(33.0)$ & $38(31.0)$ & $79(32.0)$ \\
Gestation Age (weeks) & & & \\
$<28$ & $3(2.40)$ & $1(1.00)$ & $4(2.00)$ \\
$28-37$ & $52(42.3)$ & $54(44.0)$ & $106(43.0)$ \\
$\geq 37$ & $68(55.3)$ & $68(53.0)$ & $136(55.0)$ \\
Delivery & & & \\
Delivered & $83(68.0)$ & $86(70.0)$ & $169(69.0)$ \\
Undelivered & $40(32.0)$ & $37(30.0)$ & $77(31.0)$ \\
\hline
\end{tabular}

Table 2. The proportion of women presenting with symptoms of or similar to imminent eclampsia during the index pregnancy. Data presented as n (\%).

\begin{tabular}{|c|c|c|c|}
\hline Characteristic & $\begin{array}{c}\text { Eclamptics } \\
n=123\end{array}$ & $\begin{array}{c}\text { Normotensive } \\
\mathrm{n}=123\end{array}$ & p value \\
\hline \multicolumn{4}{|l|}{ Headache } \\
\hline Yes & $108(88.0)$ & $53(43.0)$ & $<0.001$ \\
\hline No & $15(12.0)$ & $70(57.0)$ & \\
\hline \multicolumn{4}{|l|}{ Visual problem } \\
\hline Yes & $48(39.0)$ & $4(3.00)$ & $<0.001$ \\
\hline No & $75(61.0)$ & $119(97.0)$ & \\
\hline \multicolumn{4}{|l|}{ Abdominal pain } \\
\hline Yes & $58(47.0)$ & $47(38.0)$ & 0.156 \\
\hline No & $65(53.0)$ & $76(62.0)$ & \\
\hline \multicolumn{4}{|l|}{ Nausea } \\
\hline Yes & $74(60.0)$ & $66(54.0)$ & 0.303 \\
\hline No & $49(40.0)$ & $57(46.0)$ & \\
\hline \multicolumn{4}{|l|}{ Vomiting } \\
\hline Yes & $76(62.0)$ & $71(58.0)$ & 0.516 \\
\hline No & $47(38.0)$ & $52(42.0)$ & \\
\hline \multicolumn{4}{|c|}{ One or more symptom ${ }^{a}$} \\
\hline Yes & $111(90.0)$ & $66(54.0)$ & $<0.001$ \\
\hline No & $12(10.0)$ & $57(46.0)$ & \\
\hline
\end{tabular}

${ }^{\mathrm{a}}$ Headache, visual problems, abdominal pain and nausea/vomiting. 
group [(88\% vs 43\%) and (39\% vs 3\%) respectively].

Eclamptic headache was characteristically more severe among cases with a mean score of 2.07 ( \pm 0.99 SD) compared with $0.65( \pm 0.85 \mathrm{SD}),(95 \% \mathrm{CI}: 1.18-1.65)$ for referents. As can be seen in Table 3, 46.3\% of the cases reported severe headache compared to $5.7 \%$ of the referent group. The location of headache among eclamptic women was mainly frontal $(65.7 \%)$ in contrast to frontal $(41.5 \%)$ or generalized locations $(39.6 \%)$ for the referent women.

Table 3. Characteristics of symptoms as experienced by eclamptic and normotensive women during the index pregnancy. Data presented as $\mathrm{n}(\%)$.

\begin{tabular}{|c|c|c|c|}
\hline Characteristic & $\begin{array}{l}\text { Eclamptics } \\
n=108\end{array}$ & $\begin{array}{c}\text { Normotensive } \\
\quad n=53\end{array}$ & $p$ value \\
\hline \multicolumn{4}{|l|}{ Headache severity } \\
\hline Mild & $12(11.1)$ & $29(54.7)$ & \\
\hline Moderate & $46(42.6)$ & $21(39.6)$ & $<0.001$ \\
\hline Severe & $50(46.3)$ & $3(5.70)$ & \\
\hline \multicolumn{4}{|l|}{ Site of headache } \\
\hline Frontal & $71(65.7)$ & $22(41.5)$ & \\
\hline Occipital & $4(3.70)$ & $1(1.90)$ & \\
\hline Parietal & $11(10.2)$ & $7(13.2)$ & 0.01 \\
\hline Vertex & $5(4.60)$ & $2(3.80)$ & \\
\hline Generalized & $17(15.7)$ & $21(39.6)$ & \\
\hline \multicolumn{4}{|c|}{ Site of abdominal pain } \\
\hline Upper & $21(36.2)$ & $4(8.50)$ & \\
\hline Lower & $32(55.2)$ & $26(55.3)$ & $<0.001$ \\
\hline General & $5(8.60)$ & $17(36.2)$ & \\
\hline \multicolumn{4}{|l|}{ Type of pain } \\
\hline Dull aching & $12(20.7)$ & $5(10.6)$ & \\
\hline Colicky & $21(36.2)$ & $17(36.2)$ & \\
\hline Cramping & $8(13.8)$ & $16(34.0)$ & $<0.321$ \\
\hline Burning & $7(12.1)$ & $2(4.30)$ & \\
\hline Other & $10(17.2)$ & $7(14.9)$ & \\
\hline \multicolumn{4}{|l|}{ Severity of nausea } \\
\hline Non severe & $44(60.0)$ & $45(68.0)$ & \\
\hline Severe & $20(27.0)$ & $15(23.0)$ & 0.529 \\
\hline Very severe & $10(13.0)$ & $6(9.00)$ & \\
\hline \multicolumn{4}{|l|}{ Type of vomiting } \\
\hline Projectile & $4(5.00)$ & $0(0.00)$ & 0.121 \\
\hline Non projectile & $72(95.0)$ & $71(100.0)$ & \\
\hline
\end{tabular}

Very few (3\%) normotensive women reported visual problems (Table 3). Regarding eclamptic women who presented with visual problems, the complaints were blurring of vision $(94 \%)$, blind spots $(67 \%)$, photophobia (21\%) and total blindness (15\%).

Although abdominal pain was commonly reported by both the cases and referent women, upper right quadrant abdominal pain was significantly more reported by eclamptic women $(36.2 \%)$ than the normotensive $(8.5 \%)$, $\mathrm{p}=0.001$. Nausea and vomiting were not significantly different in occurrence, severity and character among the two groups.

Among eclamptic women who presented with headache $(89 \%)$ or abdominal pain $(71 \%)$, fits occurred within 7 days of the symptom. Almost all mothers (98\%) who reported visual problems had fits within 12 hours. Most cases of nausea (63\%) and vomiting (62\%) preceded eclamptic fits by more than 7 days (Table 4).

\section{DISCUSSION}

Eclampsia continues to lead as a cause of maternal deaths despite the availability of effective prophylactic treatment. The clinical challenge lies in predicting which women with a diagnosis of pre-eclampsia will soon progress into eclampsia. Headache, visual disturbances, abdominal pain, nausea, and vomiting are the most consistent prodromal symptoms of eclampsia. These symptoms have the potential to alert health service providers on

Table 4. Time elapse from onset of symptoms to development of fits among eclamptic women.

\begin{tabular}{ccc}
\hline Characteristic & Number & Percent \\
\hline Headache $(\mathbf{n}=\mathbf{1 0 8})$ & 96 & 89.0 \\
$0-7$ days & 12 & 11.0 \\
$>7$ days & & \\
Abdominal pain $(\mathbf{n}=\mathbf{5 8})$ & 41 & 71.0 \\
$0-7$ days & 17 & 29.0 \\
$>7$ days & & \\
Nausea $(\mathbf{n}=\mathbf{7 4})$ & 27 & 37.0 \\
$0-7$ days & 47 & 63.0 \\
$>7$ days & & 38.0 \\
Vomiting $(\mathbf{n}=\mathbf{7 6})$ & 29 & 62.0 \\
$0-7$ days & 47 & 98.0 \\
$>7$ days & & 2.00 \\
Visual problems $(\mathbf{n}=\mathbf{4 8})$ & 47 & \\
$0-12$ hours & & \\
$>12$ hours & & \\
\end{tabular}


which patient is most likely to benefit from prophylactic treatment for eclampsia while minimizing potentially harmful interventions and cost [24,25]. Although the ability of these symptoms to predict adverse maternal outcomes is supported by some researchers $[21,25]$ it has been questioned by others [27]. Moreover, similar symptoms are common among normal pregnant mothers and can be confused with symptoms caused by tropical infections such as malaria [8,28-31]. With this background it was imperative to attempt to characterize the prodromal symptoms and distinguish them from similar symptoms usually experienced by normotensive women in pregnancy.

We found that prodromal symptoms occurred in $90 \%$ of eclamptic women which is within the $41 \%$ to $91 \%$ range commonly reported in literature $[24,25,37,38]$. In the current study, among the prodromal symptoms, headache was the most experienced $(88 \%)$ by eclamptic women which is comparable with the $81 \%$ incidence in a recent study of eclamptic women in Nothern Tanzania [24]. In addition, our study proves that headache is significantly commoner among eclamptic than non eclamptic pregnant women.

Headache and visual disturbance are recognized neurological manifestations of severe pre-eclampsia that share a common pathophysiological base [22,26,31]. It was therefore not surprising to note that visual disturbance was the second most frequent prodromal symptom in the current study-a pattern which has been reported by others [24,34,39]. Among eclamptic women headache was characteristically frontal and severe with the first eclamptic fit occurring within one week of its onset in contrast to headache among the normotensive women in whom it was frontal or generalized and mild in severity. There are few studies that have described prodromal symptoms of eclampsia. Katz and colleagues in USA noted that eclamptic mothers described headache as "the worst headache of their lives" [26,31]. Frontal headache in relation with eclampsia has also been reported by some [18], but not supported by others who variably describe it as bitemporal, occipital, diffuse or only occasionally frontal $[26,31]$. It is unclear whether the difference is a true variation among study populations or it is due to study biases. Further studies are needed to clarify this difference.

Visual problems were reported by two fifth of all eclamptic women with blurring of vision being the most common presentation, followed by blind spots, photophobia and total blindness. Although the incidence of visual problems ranked second to headache, it is the most ominous symptom as in almost all eclamptic women fits occurred within 12 hours of its onset. This is probably because the changes like brain oedema, microvascular thrombosis and necrosis that cause visual symptoms would almost immediately lead to epileptic fits [22]. In support to this notion, visual problems were rarely reported in normotensive mothers with only 4 women presented with visual complaints.

The presentation with upper right quadrant abdominal pain, was statistically significantly higher in eclamptics than normotensive mothers probably due to its linkage with liver pathophysiological changes in severe preeclampsia. Upper abdominal pain, as is the case with nausea and vomiting, are all linked with liver injury [17, $21,25,26]$ but the importance of these symptoms in predicting eclampsia has been unsatisfactory due to low sensitivity. Other types of abdominal pain, nausea and vomiting were largely indistinguishable from similar symptom experiences by normotensive mothers. This implies that the symptoms represent a more heterogeneous group caused by a variety of factors.

The interpretation of our findings is limited in that the patients' account on prodromal symptoms was retrospective hence subject to recall bias. However, a short duration for the symptoms (during index pregnancy) and the design to interview the mothers immediately after an eclamptic fit should have minimized such a bias. Our decision to interview eclamptic mothers rather than reliance on the case note records is one of the strengths of this study in contrast to similar studies that have used retrospective data [21,25-26]. The characteristics of the prodromal symptoms established by this study could be used to develop a prediction model for eclampsia in the process to improve management of pre-eclamptic patients and prevention of eclampsia.

In conclusion, headache, abdominal pain, nausea and vomiting are common during pregnancy whether or not complicated by eclampsia but visual disturbances were not as common in normotensive mothers. The characteristics of headache and visual disturbances can be reasonably distinguished among eclamptics and normotensive women. Visual disturbance is the most ominous for occurrence of eclamptic fit within twelve hours. General abdominal pain, nausea and vomiting are heterogeneous and not distinguishable among eclamptic and normotensive women.

\section{ACKNOWLEDGEMENTS}

The authors gratefully acknowledge the cooperation of the participant mothers, and members of academic staff in the department of Obstetrics and Gynecology (MUHAS) for useful inputs during proposal development and MNH administration for permission to conduct the study.

\section{REFERENCES}

[1] Sibai, B.M. (2004) Pre-eclampsia: An inflammatory syndrome? American Journal of Obstetrics and Gynecology, 
191, 1061-1062. doi:10.1016/j.ajog.2004.03.042

[2] Sibai, B.M., Caritis, S. and Hauth, J. (2003) What we have learned about pre-eclampsia. Semin in Perinatology, 27, 239-246. doi:10.1016/S0146-0005(03)00022-3

[3] Shneider, H. (1994) Liver pathology and HELLP syndrome. Archives of Gynecology and Obstetrics, 255, 245254.

[4] Williams, K., Bianco Lim, K., Wilson, S., et al. (2004) Can clinical symptomology predict maternal cerebral perfusion pressure in pre-eclampsia? Revista Chilena de Obstetrica y Ginecologia, 69, 361-367.

[5] Belfort, M.A., Clark, S.L. and Sibai, B. (2006) Cerebral hemodynamics in pre-eclampsia: Cerebral perfusion and the rationale for an alternative to magnesium sulfate. $\mathrm{Ob}$ stetrical \& Gynecological Survey, 61, 655-665. doi:10.1097/01.ogx.0000238670.29492.84

[6] Mulla, Z.D., Gonzalez-Sanchez, J.L. and Nuwayhid, B.S. (2007) Descriptive and clinical epidemiology of preeclampsia and eclampsia in Florida. Ethnicity \& Disease, 17, 736-741.

[7] Saftlas, A.F., Olson, D.R., Franks, A.L., Atrash, H.K. and Pokras, R. (1990) Epidemiology of pre-eclampsia and eclampsia in the United States, 1979-1986. American Journal of Obstetrics and Gynecology, 163, 460-465.

[8] McGready, R. and Nosten, F. (2008) Symptomatic malaria in pregnancy. Journal of Obstetrics and Gynaecology, 28, 463. doi:10.1080/01443610802166131

[9] Duley, L. (2009) The global impact of pre-eclampsia and eclampsia. Seminars in Perinatology, 33, 130-137. doi:10.1053/j.semperi.2009.02.010

[10] Moodley, J. (2010) Maternal deaths associated with eclampsia in South Africa: Lessons to learn from the confidential enquiries into maternal deaths, 2005-2007. South African Medical Journal, 100, 717-719.

[11] Vigil-De Gracia, P. (2009) Maternal deaths due to eclampsia and HELLP syndrome. International Journal of Gynecology \& Obstetrics, 104, 90-94. doi:10.1016/j.ijgo.2008.09.014

[12] Langer, A., Villar, J., Tell, K., Kim, T. and Kennedy, S. (2008) Reducing eclampsia-related deaths-A call to action. The Lancet, 371, 705-706. doi:10.1016/S0140-6736(08)60321-9

[13] Von Dadelszen, P., Payne, B., Li, J., Ansermino, J.M., Broughton Pipkin, F., Cote, A.M., et al. (2011) Prediction of adverse maternal outcomes in pre-eclampsia: Development and validation of the fullPIERS model. The Lancet, 377, 219-227. doi:10.1016/S0140-6736(10)61351-7

[14] Urassa, D.P., Carlstedt, A., Nystrom, L., Massawe, S.N. and Lindmark, G. (2006) Eclampsia in Dar es Salaam, Tanzania-Incidence, outcome, and the role of antenatal care. Acta Obstetrica et Gynecologica Scandinavica, 85, 571-578. doi:10.1080/00016340600604880

[15] Kidanto, H.L., Mogren, I., Massawe, S.N., Lindmark, G. and Nystrom, L. (2009) Criteria-based audit on management of eclampsia patients at a tertiary hospital in Dar es Salaam, Tanzania. BMC Pregnancy Childbirth, 9, 13. doi:10.1186/1471-2393-9-13

[16] Muganyizi, P.S. and Shagdara, M.S. (2011) Predictors of extra care among magnesium sulphate treated eclamptic patients at Muhimbili National Hospital, Tanzania. BMC Pregnancy \& Childbirth, 11, 41. doi:10.1186/1471-2393-11-41

[17] Dekker, G.A. and Sibai, B.M. (1998) Etiology and pathogenesis of pre-eclampsia: Current concepts. American Journal of Obstetrics \& Gynecology, 179, 1359-1375. doi:10.1016/S0002-9378(98)70160-7

[18] Menzies, J., Magee, L.A., Macnab, Y.C., Ansermino, J.M., Li, J., Douglas, M.J., et al. (2007) Current CHS and NHBPEP criteria for severe pre-eclampsia do not uniformly predict adverse maternal or perinatal outcomes. Hypertension in Pregnancy, 26, 447-462. doi:10.1080/10641950701521742

[19] Thangaratinam, S., Coomarasamy, A., O’Mahony, F., Sharp, S., Zamora, J., Khan, K.S., et al. (2009) Estimation of proteinuria as a predictor of complications of pre-eclampsia: A systematic review. BMC Medine, 7, 10. doi:10.1186/1741-7015-7-10

[20] Thangaratinam, S., Ismail, K.M., Sharp, S., Coomara-samy, A. and Khan, K.S. (2006) Accuracy of serum uric acid in predicting complications of pre-eclampsia: A systematic review. BJOG: An International Journal of Obstetrics and Gynaecology, 113, 369-378. doi:10.1111/j.1471-0528.2006.00908.x

[21] Thangaratinam, S., Langenveld, J., Mol, B.W. and Khan, K.S. (2011) Prediction and primary prevention of preeclampsia. Best Practice \& Research Clinical Obstetrics \& Gynaecology, 25, 419-433. doi:10.1016/i.bpobgyn.2011.02.008

[22] Matsuda, H., Sakaguchi, K., Shibasaki, T., Takahashi, H., Kawakami, Y., Furuya, K., et al. (2005) Cerebral edema on MRI in severe preeclamptic women developing eclampsia. Journal of Perinatal Medicine, 33, 199-205. doi:10.1515/JPM.2005.037

[23] Kozic, J.R., Benton, S.J., Hutcheon, J.A., Payne, B.A., Magee, L.A. and von Dadelszen, P. (2011) Abnormal liver function tests as predictors of adverse maternal outcomes in women with pre-eclampsia. Journal of Obstetrics and Gynaecology Canada, 33, 995-1004.

[24] Cooray, S.D., Edmonds, S.M., Tong, S., Samarasekera, S.P. and Whitehead, C.L. (2011) Characterization of symptoms immediately preceding eclampsia. Obstetrics \& Gynecology, 118, 995-999. doi:10.1097/AOG.0b013e3182324570

[25] Thangaratinam, S., Gallos, I.D., Meah, N., Usman, S., Ismail, K.M. and Khan, K.S. (2011) How accurate are maternal symptoms in predicting impending complications in women with pre-eclampsia? A systematic review and meta-analysis. Acta Obstetricia et Gynecologica Scandinavica, 90, 564-573. doi:10.1111/j.1600-0412.2011.01111.x

[26] Katz, V.L., Farmer, R. and Kuller, J.A. (2000) Preeclampsia into eclampsia: Toward a new paradigm. American Journal of Obstetrics \& Gynecology, 182, 13891396. doi: $10.1067 / \mathrm{mob} .2000 .106178$

[27] Yen, T.W., Payne, B., Qu, Z., Hutcheon, J.A., Lee, T., Magee, L.A., et al. (2011) Using clinical symptoms to predict adverse maternal and perinatal outcomes in wo- 
men with pre-eclampsia: Data from the PIERS (Preeclampsia Integrated Estimate of Risk) study. Journal of Obstetrics and Gynaecology Canada, 33, 803-809.

[28] Kvisvik, E.V., Stovner, L.J., Helde, G., Bovim, G. and Linde, M. (2011) Headache and migraine during pregnancy and puerperium: The MIGRA-study. The Journal of Headache and Pain, 12, 443-451. doi:10.1007/s10194-011-0329-1

[29] Aegidius, K., Zwart, J.A., Hagen, K. and Stovner, L. (2009) The effect of pregnancy and parity on headache prevalence: The Head-HUNT study. Headache: The Journal of Head and Facec Pain, 49, 851-859. doi:10.1111/j.1526-4610.2009.01438.x

[30] Naumann, C.R., Zelig, C., Napolitano, P.G. and Ko, C.W. (2012) Nausea, Vomiting, and Heartburn in Pregnancy: A Prospective Look at Risk, Treatment, and Outcome. The Journal of Maternal-Fetal \& Neonatal Medicine, 25, 1488-1493. doi:10.3109/14767058.2011.644363

[31] Dixit, A., Bhardwaj, M. and Sharma, B. (2012) Headache in pregnancy: A nuisance or a new sense? Obstetrics and Gynecology International, 2012, 697697.

[32] Fleiss, J.L. (1981) Statistical methods for rates and proportions. 2nd Edition, John Wiley, New York.

[33] Lines, C.R., Vandormael, K. and Malbecq, W. (2001) A comparison of visual analog scale and categorical ratings of headache pain in a randomized controlled clinical trial with migraine patients. Pain, 93, 185-190.
doi:10.1016/S0304-3959(01)00315-3

[34] Harms, E., Bahr, M. and Klock, F.K. (1991) The HELPP syndrome-A severe complication of pre-eclampsia. A presentation of 19 cases from 1983 to 1990. Zeitschrift für Geburtshilfe und Perinatologie, 195, 187-192.

[35] Witlin, A.G., et al. (1999) Risk factors for abruptio placentae and eclampsia: Analysis of 445 consecutively managed women with severe pre-eclampsia and eclampsia. American Journal of Obstetrics \& Gynecology, 180, 1322 1329. doi:10.1016/S0002-9378(99)70014-1

[36] Black, K.D. (2007) Stress, symptoms, self-monitoring confidence, well-being, and social support in the progression of pre-eclampsia/gestational hypertension. Journal of Obstetric, Gynecologic, \& Neonatal Nursing, 36, 419429. doi:10.1111/j.1552-6909.2007.00173.x

[37] Douglas, K.A. and Redman, C.W. (1994) Eclampsia in the United Kingdom. British Medical Journal, 309, 13951400. doi:10.1136/bmj.309.6966.1395

[38] Mahmoudi, N., et al. (1999) Eclampsia: A 13-year experience at a United States tertiary care center. Journal of Women's Health \& Gend-Based Medicine, 8, 495-500. doi:10.1089/jwh.1.1999.8.495

[39] Ben Salem, F., et al. (2003) Risk factors for eclampsia: A case-control study. Annales Francaise D'Anesthesie et de Reanimation, 22, 865-869.

doi:10.1016/j.annfar.2003.08.006 